\title{
Aterectomía rotacional y recuperación exitosa de fragmento de alambre guía roto
}

\author{
Rotational atherectomy and successful retrieval of broken guidewire \\ fragment
}

Jorge N. Wisner', Marcel G. Voos Budal Arins', Alejandro Tettamanzi ${ }^{1}$

\section{RESUMEN}

La aterectomía rotacional es la técnica ideal para tratar las estenosis calcificadas no dilatables. La fractura de un alambre guía en una arteria coronaria es una complicación poco frecuente de la angioplastia coronaria. Presentamos un caso clínico de un paciente con estenosis severa no dilatable de arteria circunfleja que en un intento previo de angioplastia presentó como complicación la fractura de un alambre guía coronario que fue dejado en el lecho distal del vaso y fue resuelto con aterectomía rotacional seguido de implante de stent con recuperación exitosa del fragmento de alambre guía coronario con lazo.

Palabras claves: aterectomía rotational, angioplastia, complicación, cuerpo extraño.

\begin{abstract}
The rotational atherectomy is the ideal technique to treat undilatable calcified stenosis. Fractured coronary guidewire is a relatively uncommon complication of percutaneous coronary intervention. We report a clinical case of a patient with undilatable severe stenosis of left circunflex artery who in an unsuccessful attempt of percutaneous coronary intervention presented as complication fracture of a coronary guidewire left in the distal vessel. Rotational atherectomy followed by stent deployment and successful retrieval with a snare of broken guidewire fragment was made.
\end{abstract}

Key words: rotational atherectomy, angioplasty, complication, foreign body.

Revista Argentina de Cardioangiología Intervencionista 2014;5(2):144-146

\section{INTRODUCCIÓN}

Actualmente, la indicación principal de la aterectomía rotacional son las lesiones calcificadas indilatables o no penetrables con balón, como procedimiento previo a la angioplastia con stent, las lesiones ostiales y las oclusiones totales crónicas. En estos grupos, diferentes estudios han demostrado que el uso combinado de aterectomía rotacional con stent puede aumentar el éxito primario y reducir la tasa de eventos adversos. ${ }^{1}$

La fractura de un alambre guía en una arteria coronaria es una complicación poco frecuente de la angioplastia coronaria. Varios métodos fueron descriptos para la recuperación de estos fragmentos retenidos en la circulación coronaria. En las arterias coronarias, los lazos capturan la mayoría de estos fragmentos de alambre guía. ${ }^{2,3}$ Presentamos un caso clínico de un paciente con estenosis severa no dilatable de arteria circun-

\footnotetext{
Servicio de Hemodinamia y Cardiología Intervencionista. Hospital Universitario CEMIC. Argentina.

$\triangle$ Correspondencia: Marcel G. Voos Budal Arins. Avenida Triunvirato 4355 Piso $5^{\circ}$ Dto B. (1431)CABA, Argentina| marcelvoos@gmail.com
}

Conflictos de intereses: no existen.

Recibido: 20-11-2013|Aceptado: 2-1-2014. fleja que en un intento previo de angioplastia presentó como complicación la fractura de un alambre guía coronario que fue dejado en el lecho distal del vaso y fue resuelto con aterectomía rotacional seguido de implante de stent con recuperación exitosa del fragmento de alambre guía coronario con lazo.

\section{REPORTE DEL CASO}

Paciente masculino de 56 años, tabaquista, sin antecedentes patológicos de relevancia que ingresa a otra institución por infarto agudo de miocardio con supradesnivel del segmento ST en cara anterior, realizándose angioplastia transluminal coronaria (ATC) exitosa con dos stents convencionales en tercio proximal de arteria descendente anterior (DA) por oclusión trombótica de esta. La coronariografía (CCG) también evidenció la presencia de una estenosis severa y calcificada en tercio medio de arteria circunfleja $(\mathrm{Cx})$ y arteria coronaria derecha $(\mathrm{CD}) \sin$ obstrucciones significativas. Luego de cuatro días del procedimiento inicial, se intentó realizar ATC de Cx que fue fallida por la presencia de una estenosis severa y calcificada, indilatable por balón. En el intento de realizar el procedimiento, la punta del alambre guía 0,014 " se fracturó y fue abandonado en el lecho distal del ramo marginal obtuso (RMO). Tres días después del intento fallido de ATC de Cx, el paciente fue derivado a nuestra 


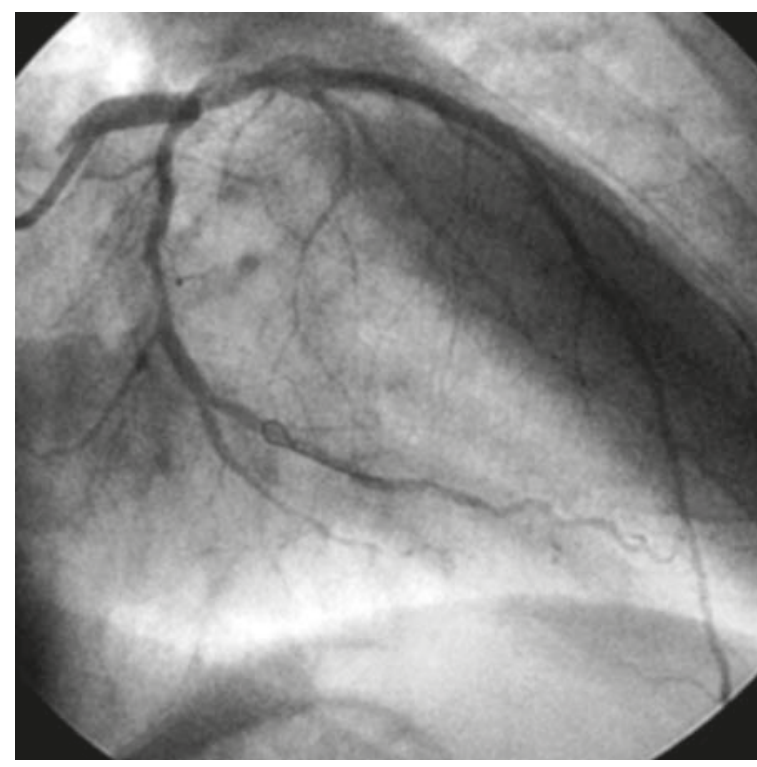

Figura 1.

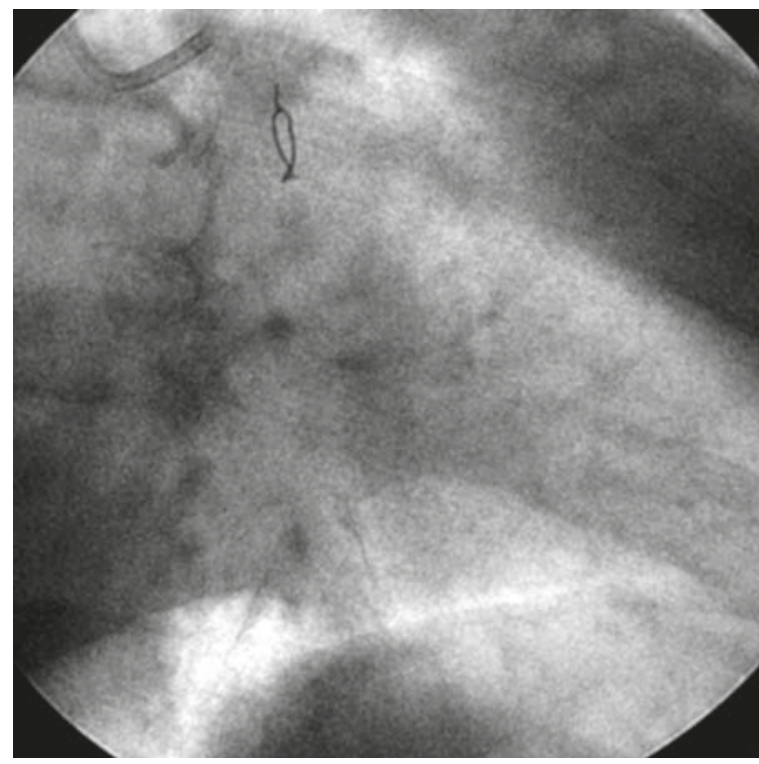

Figura 3.

institución para la realización de aterectomía rotacional (AR) y recuperación del fragmento de alambre guía. $\mathrm{Al}$ ingreso a nuestra institución el paciente se encontraba asintomático y sin signos de insuficiencia cardíaca. El electrocardiograma muestra ondas $\mathrm{T}$ negativas en $\mathrm{DI}, \mathrm{aVL}$, V2-V6. El ecocardiograma reveló hipoquinesia apical con fracción de eyección de 57\%. troponina T ultrasensible 237 ng/l (valor de referencia 14 ng/l). Se realizó hidratación con solución fisiológica 24 horas antes del procedimiento para prevenir nefropatía inducida por contraste.

La CCG realizada en nuestro servicio evidenció tronco de arteria coronaria izquierda (TCI) sin obstrucciones, DA sin obstrucciones significativas con dos stents en tercio proximal, $\mathrm{Cx}$ con estenosis severa y calcificada en tercio medio con fragmento de alambre guía 0,014" en el lecho distal del RMO (Figura 1), CD sin obstrucciones significativas.

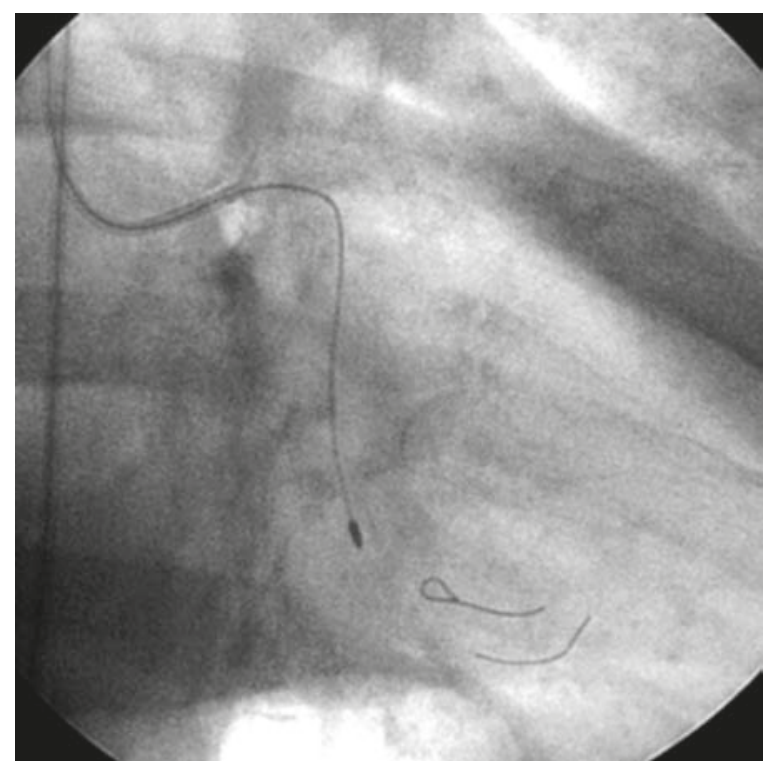

Figura 2.

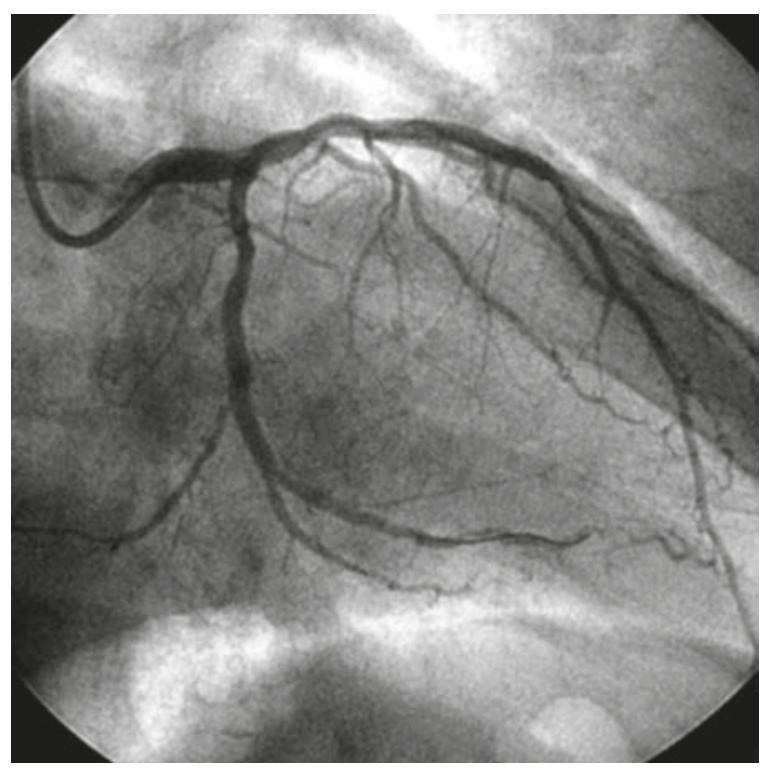

Figura 4.

El TCI fue cateterizado con un catéter guía XB 3.56 Fr por acceso femoral derecho. Se progresó un alambre guía 0.009" (Rotawire) por la CX hasta el lecho distal del ramo posteroventricular, se realizó AR (Rotablator-Boston Scientific) con una oliva de $1.5 \mathrm{~mm}$ de diámetro a $180.000 \mathrm{rpm}$ (Figura 2) seguido de dilatación con un balón no-complaciente de $2,5 \times 20 \mathrm{~mm}$ (Quantum Maverick- Boston Scientific) hasta 14 atm obteniéndose dilatación exitosa de la lesión. Luego se progresa el alambre guía 0,009" hasta el lecho distal del RMO y se avanza un lazo de $2 \mathrm{~mm}$ de diámetro (Amplatz GooseNeck Microsnare Kit - eV3) distal al fragmento de alambre guía 0,014". El fragmento roto de alambre guía 0,014 " fue recuperado exitosamente con el lazo (Figura 3). Finalmente, se implanta un stent de cromo-platino 3,0 $\times 20 \mathrm{~mm}$ (OMEGA - Boston Scientific) que fue impactado a 14 atm en ter- 
cio medio de $\mathrm{Cx}$ con buen resultado angiográfico (Figura 4). El paciente fue enviado a Unidad Coronaria por 24 horas y fue dado de alta 2 días después del procedimiento sin complicaciones.

\section{DISCUSIÓN}

La AR es la técnica ideal para tratar las estenosis calcificadas indilatables, cambiando la compliance de la lesión, minimizando el trauma vascular, creando un diámetro luminal mínimo mayor que permite el implante de stents en lesiones complejas. ${ }^{4}$

Este caso clínico describe la remoción exitosa de un fragmento roto de la punta de un alambre guía 0,014 " retenido en la arteria coronaria. Muchos casos de frac-

\section{BIBLIOGRAFÍA}

1. Hoffmann R, MintzGS, KentKM, Pichard AD, SatlerLF, Popma JJ, etal.Comparative early and nine-month results of rotational atherectomy, stents, and the combination of both for calcified lesions in large coronary arteries. J Am Coll Cardiol 1998 Mar 1;81(5):552-557.

2. Hartzler GO, Rutherford BD, McConahay DR. Retained percutaneous transluminal coronary angioplasty equipment components and their management. Am J Cardiol 1987;60:1260-1264.

3. Krone RJ. Sucsessful percutaneous removal of retained broken coronary angioplasty guidewire. Cathet Cardiovasc Diagn 1986;12:409-410.

4. Buchbinder M, Fortuna R, Sharma SK, Bass T, Kipperman R, Greenberg JD. tura se producen cuando el extremo distal del alambre guía queda atrapado en la arteria coronaria. Un fragmento de alambre guía retenido en la circulación puede pasar inadvertido sin secuelas o puede causar complicaciones como trombosis, embolia y sepsis. ${ }^{5-7}$ Los informes de extracción de alambres guía fracturados son un testimonio del ingenio y la creatividad de los intervencionistas, debido a que existen numerosos métodos descriptos para la realización del procedimiento. En este caso, como el fragmento se encontraba totalmente en lecho distal de la arteria coronaria, la utilización de un lazo pequeño demostró ser la técnica más exitosa. En conclusión, nos enfrentamos con un caso muy desafiante que requirió del empleo de varias técnicas complejas para su resolución sin complicaciones.

Debulking prior to stenting improves acute outcomes: Early results from the SPORT Trial. J Am Coll Cardiol 2000;35(suppl A):8.

5. Gavlick K, Blankenship JC. Snare retrieval of the distal tip of a fractured rotational atherectomy guidewire: Roping the steers by its horns. I Invasive Cardiol. 2005 Dec;17(12):E55-58.

6. Lotan C, Hasin Y, Stone D, etal. Guidewireentrapment during PTCA:A potentially dangerous complication. Cathet Cardiovasc Diagn 1987;13:309-312.

7. Vrolix M, VanhaeckeJ, Piessens J, Geest HD. An unusual case of guide wire fracture during percutaneous trans/uminal coronary angioplasty. Cathet Cardiovasc Diagn 1988;15:99-102. 